\title{
Performance Analysis of Multi-Parametric Call Admission Control Strategies in Un-Buffered Multi-Service Cellular Wireless Networks
}

\author{
Jang Hyun Baek ${ }^{1}$, Che Soong Kim ${ }^{2}$, Agassi Melikov ${ }^{3}$, Mehriban Fattakhova ${ }^{3}$ \\ ${ }^{1}$ Department of Industrial and Information Systems Engineering, Chonbuk National University, Jeonju, Republic of Korea \\ ${ }^{2}$ Department of Industrial Engineering, Sangji University, Wonju, Republic of Korea \\ ${ }^{3}$ Department of Aerospace Information Technologies \& Control Systems, National Aviation Academy, Azerbaijan \\ E-mail:jbaek@chonbuk.ac.kr,dowoo@sangji.ac.kr,\{agassi.melikov,meri-fattax\}@rambler.ru \\ Received December 28, 2009; revised January 12, 2010; accepted January 15, 2010
}

\begin{abstract}
In this paper model of integrated voice/data cellular wireless networks $(\mathrm{CWN})$ are investigated. The unified approximate approach to calculate the desired Quality of Service (QoS) metrics in an isolated cell of such networks under two multi-parametric call admission control (CAC) strategies is developed. One of them is based on the guard channels scheme while the second is based on a threshold scheme. Results of the numerical experiments are given and a comparison of QoS metrics under different CAC strategies is carried out.
\end{abstract}

Keywords: Cellular Networks, Call Admission Control, Quality of Service, Calculation Algorithm

\section{Introduction}

A cellular wireless network (CWN) consists of radio access points, called base stations (BS), each covering a certain geographic area. With distance, the power of radio signals fade away (fading or attenuation of signal occurs) which makes it possible to use the same frequencies over several cells, but in order to avoid interference, this process must be carefully planned. For better use of frequency resources, existing carrier frequencies are grouped, and the number of cells, in which this group of frequencies is used, defines the so called, frequency reuse factor. Therefore, in densely populated areas with a large number of mobile subscribers (MS), small dimensioned cells (micro-cells and pico-cells) are to be used.

In connection with the limitation of transmission spectrum in a CWN, problems of allocation of common spectrum among cells are very important. A unit of the wireless spectrum, necessary for serving a single user is called a channel (for instance, time slots in TDMA are considered as channels). There are three solutions for the channels allocation problem: Fixed Channel Allocation (FCA), Dynamic Channel Allocation (DCA) and Hybrid Channel Allocation (HCA). Advantages and disadvantage of each of these are well known [1-3]. At the same time, owing to realization simplicity, the FCA scheme is widely used in existing cellular networks. In this paper models with FCA schemes are considered.

Quality of service (QoS) in the certain cell with the FCA scheme could be improved if appropriate call ad-mission control (CAC) strategies for the heterogeneous traffic are provided. The use of such an access strategy doesn't require many resources, therefore this method could be considered operative and more effective in view of problems relating to resource shortages.

Apart from original (or new) calls (o-calls) flows, additional classes of calls that require a special approach also exist in wireless cellular networks. These are socalled handover calls (h-calls). This is specific only for wireless cellular networks. The essence of this phenomenon is that a moving MS, that already established connection with a network, passes boundaries between cells and gets served by a new cell. From the new cell's point of view this is an h-call, and since the connection with the MS has already been established, the handling of the transfer to a new cell must be transparent for the user. In other words, in wireless networks the call may occupy channels from different cells, several times during the duration of the call. This means that the channel occupation period is not the same as the call duration.

Mathematical models of call handling processes in multi-service $\mathrm{CWN}$ can be developed adequately enough 
based on a queuing theory of networks with different types of calls and random topology. Such models are researched poorly in literature [4-6]. This is explained by the fact, that despite the elegance of those models, in practice they are useful only for small dimensional networks and with some limiting simplifying assumptions that are contrary to fact in real functioning wireless networks. In connection with that, in the majority of the research work models of an isolated cell are analyzed.

In the overwhelming majority of available work, one-dimensional queuing models of call handling processes in an isolated cell of mono-service CWN are proposed. However these models cannot describe the studying processes in multi-service $\mathrm{CWN}$ since in such net-works calls of heterogeneous traffic differ significantly with respect to their bandwidth requirement and arrival rate and channel occupancy time. In connection with that in the given paper new two-dimensional (2-D) queuing models of multi-service networks are developed. In order to be specific we consider integrated voice/data CWN. In such networks voice calls (v-calls) are more susceptible to possible losses and delays than data (original or handover) call (d-calls). That is why a number of different CAC strategies for prioritization of v-calls are suggested in various works, mostly implying the use of guard channels (cutoff strategy) for high priority calls [7-8] and/or threshold strategies [9] which restrict the number of low priority calls in channels.

In this paper we introduce a unified approach to approximate performance analysis of two multi-parametric $\mathrm{CAC}$ in a single cell of un-buffered integrated voice/data CWN which differs from known works in this area. Our approach is based on the principles of theory of phase merging of stochastic systems [10].

The proposed approach allows overcoming an assumption made in almost all of the known papers about equality of handling intensities of heterogeneous calls. Due to this assumption the functioning of the CWN is described with one-dimensional Markov chain (1-D MC) and authors managed simple formulas for calculating the QoS metrics of the system. However as it was mentioned in [11] the assumption of the same mean channel occupancy time even for both original and handover calls of the same class is unrealistic. The presented models are more general in terms of handling intensities and the equality is no longer required.

This paper is organized as follows. In Section 2, we provide a simple algorithm to calculate approximate values of desired QoS metrics of the model of integrated voice/data networks under $\mathrm{CAC}$ based on the guard channels strategy. A similar algorithm is suggested in Section 3 for the same model under CAC based on a threshold strategy. In Section 4, we give results of numerical experiments which indicate high accuracy of the proposed approximate algorithms as well as a comparison of QoS metrics in different CAC strategies. In Sec- tion 5 we provide some conclusion remarks in conclusion.

\section{The CAC Based on Guard Channels Strategy}

It is well-known that in an integrating voice/data CWN voice calls of any type (original or handover) have a high priority over data calls and within each, flow handover calls have high priority over original calls.

As a means of assigning priorities to handover v-calls (hv-call) in such networks a back-up scheme that involves reserving a particular number of guard channels of a cell, expressly for calls of this type, is often utilized. According to this scheme any hv-call is accepted if there exists at least one free channel, while calls of the remaining kind are accepted only when the number of busy channels does not exceed some class-dependent threshold value.

We consider a model of an isolated cell in an integrated voice/data CWN without queues. This cell contains $N$ channels, $1<N<\infty$. These channels are used by Poisson flows of hv-calls, original v-calls (ov-calls), handover d-calls (hd-calls) and original d-calls (od-calls). Intensity of $x$-calls is $\lambda_{x}, x \in\{$ ov,hv,od,hd $\}$. As in almost all cited works the values of handover intensities are considered known hereinafter, although it is apparent that definition of their values depending on the intensity of original calls, shape of a cell, mobility of an MS and etc. is rather challenging and complex. However, if we consider the case of a uniform traffic distribution and at most one handover per call, the average handover intensity can be given by the ratio of the average call holding time to the average cell sojourn time [12].

To handle any narrow-band v-call (either original or handover) only one free channel is required, while one wide-band d-call (either original or handover) requires simultaneously $b \geq 1$ channels. Here it is assumed that wide-band d-calls are inelastic, i.e. all $b$ channels are occupied and released simultaneously (though can be investigated as can models with elastic d-calls).

Note that the channels' occupancy time considers both components of occupancy time: the measure of calls duration, and their mobility. Distribution functions of channel occupancy time of heterogeneous calls are assumed to be independent and exponential, but their parameters are different, namely the intensity of handling of voice (data) calls equals $\mu_{v}\left(\mu_{d}\right)$, and generally speaking $\mu_{v} \neq \mu_{d}$. If during call handling the handover procedure is initiated, the remaining handling time of this call in a new cell (yet as an h-call) is also exponentially distributed with the same mean due to the memory-free property of exponential distribution.

In a given $\mathrm{CAC}$ the procedure by which the channels are engaged by calls of different types is realized in the 
following way. As was mentioned before, if upon arrival of an hv-call, there is at least one free channel, this call seizes one of any free channels; otherwise this call is blocked. With the purpose of defining the proposed CAC for calls of other types, three parameters $N_{1}, N_{2}$ and $N_{3}$ (where $1 \leq N_{1} \leq N_{2} \leq N_{3} \leq N$ ) are introduced. It is assumed that $N_{1}$ and $N_{2}$ are multiples of $b$.

Arrived ov-call is accepted if the number of busy channels is less than $N_{3}$, otherwise it is blocked. Arrived od-call (respectively, hd-call) is accepted only in the case at most $N_{1}-b$ (respectively, $N_{2}-b$ ) busy channels, otherwise it is blocked.

Consider the problem of finding the major QoS metrics of the given multi-parametric CAC strategy - blocking (loss) probabilities of calls of each type and overall channels utilization. For simplicity of intermediate mathematical transformations first we shall assume that $b=1$. The case $b>1$ is straightforward (see below).

By adopting an assumption for the type of distribution laws governing the incoming traffics and their holding times it becomes possible to describe the operation of an isolated cell by means of a two-dimensional Markov chain (2-D MC), i.e. in a stationary regime the state of the cell at an arbitrary moment of time is described by a 2 -D vector $\boldsymbol{n}=\left(n_{d}, n_{v}\right)$, where $n_{d}$ (respectively, $\left.n_{\mathrm{v}}\right)$ is the number of data (respectively, voice) calls in the channels. Then the state space of the corresponding Markov chain describing this call handling scheme is defined as follows:

$$
S:=\left\{\boldsymbol{n}: n_{d}=0,1, \cdots, N_{2}, n_{v}=0,1, \cdots, N, n_{d}+n_{v} \leq N\right\} .
$$

Elements of generating matrix of this MC $q\left(\mathbf{n}, \mathbf{n}^{\prime}\right), \mathbf{n}, \mathbf{n}^{\prime} \in S$ are determined from the following relations:

$$
q\left(\boldsymbol{n}, \boldsymbol{n}^{\prime}\right)= \begin{cases}\lambda_{d} & \text { if } n_{d}+n_{v} \leq N_{1}-1, \boldsymbol{n}^{\prime}=\boldsymbol{n}+\boldsymbol{e}_{1}, \\ \lambda_{h d} & \text { if } N_{1} \leq n_{d}+n_{v} \leq N_{2}-1, \boldsymbol{n}^{\prime}=\boldsymbol{n}+\boldsymbol{e}_{1}, \\ \lambda_{v} & \text { if } n_{d}+n_{v} \leq N_{3}-1, \boldsymbol{n}^{\prime}=\boldsymbol{n}+\boldsymbol{e}_{2}, \\ \lambda_{h v} & \text { if } N_{3} \leq n_{d}+n_{v} \leq N-1, \boldsymbol{n}^{\prime}=\boldsymbol{n}+\boldsymbol{e}_{2}, \\ n_{d} \mu_{d} & \text { if } \boldsymbol{n}^{\prime}=\boldsymbol{n}-\boldsymbol{e}_{1}, \\ n_{v} \mu_{v} & \text { if } \boldsymbol{n}^{\prime}=\boldsymbol{n}-\boldsymbol{e}_{2}, \\ 0 & \text { in other cases, }\end{cases}
$$

where $\lambda_{d}:=\lambda_{o d}+\lambda_{h d}, \lambda_{v}:=\lambda_{o v}+\lambda_{h v}, \boldsymbol{e}_{1}=(1,0), \boldsymbol{e}_{2}=(0,1)$.

State diagram of the model and the system of global balance equations (SGBE) for the steady state probabilities $p(\boldsymbol{n}), \boldsymbol{n} \in S$ are shown in [13]. Existence of stationary regime is proved by the fact that all states of finite-dimensional state space $S$ are communicating.

Desired QoS metrics are determined via stationary distribution of the initial model. Let $P_{x}$ denote the blocking probability of the $x$-calls, $x \in\{$ hv,ov,hd,od $\}$. Then by using the PASTA theorem [14] we obtain:

$$
\begin{aligned}
P_{h v} & :=\sum_{n \in S} p(\boldsymbol{n}) \delta\left(n_{d}+n_{v}, N\right), \\
P_{o v} & :=\sum_{\boldsymbol{n} \in S} p(\boldsymbol{n}) I\left(n_{d}+n_{v} \geq N_{3}\right), \\
P_{h d} & :=\sum_{\boldsymbol{n} \in S} p(\boldsymbol{n}) I\left(n_{d}+n_{v} \geq N_{2}\right), \\
P_{o d} & :=\sum_{\boldsymbol{n} \in S} p(\boldsymbol{n}) I\left(n_{d}+n_{v} \geq N_{1}\right),
\end{aligned}
$$

where $I(A)$ denoted the indicator function of event $A$ and $\delta(i, j)$ represents Kronecker's symbols.

The mean number of busy channels $\tilde{N}$ is also calculated via stationary distribution as follows:

$$
\tilde{N}:=\sum_{k=1}^{N} k p(k),
$$

where $p(k)=\sum_{\boldsymbol{n} \in S} p(\boldsymbol{n}) \delta\left(n_{d}+n_{v}, k\right), k=\overline{1, N}$, are marginal probability mass functions.

Stationary distribution is determined as a result of the solution of an appropriate SGBE of the given 2-D MC. However, to solve the last problem one requires laborious computation efforts for large values of $N$ since the corresponding SGBE has no explicit solution. Very often the solution of such problems is evident if the corresponding 2-D MC has reversibility property [15] and hence there exists stationary distribution in a multiplicative form. Given the SGBE has a multiplicative solution only in a special case when $N_{1}=N_{2}=N_{3}=N$ (even in this case there are known computational difficulties). However, by applying Kolmogorov criteria [15] it is easily verified that the given 2-D MC is not reversible. Indeed, according to the mentioned criteria the necessary reversibility condition of 2-D MC consists in the fact that if the transition from state $(i, j)$ into state $\left(i^{\prime}, j^{\gamma}\right)$ exists, then there must also be the reverse transition from state $\left(i^{\prime}, j\right)$ to state $(i, j)$. However, for MC considered this condition is not fulfilled. So by the relations (2) in the given MC the transition $\left(n_{d}, n_{v}\right) \rightarrow\left(n_{d}-1, n_{v}\right)$ exists with intensity $n_{d} \mu_{d}$ where $n_{d}+n_{v} \geq N_{2}$, but the inverse transition not existing.

In [13], a recursive technique has been proposed as the solution to the above-mentioned SGBE. It requires multiple inversion calculations of certain matrices of sufficiently large dimensions that in itself is a complex calculating procedure. To overcome the mentioned difficulties, a new, efficient and refined approximate method for the calculation of the stationary distribution of the given model is suggested below. The proposed method, due to right selection of state space splitting of corresponding 2-D MC allows one to reduce the solution of the problem considered to calculation by explicit formulae which contain the known (even tabulated) stationary distributions of classical queuing models. 
For correct application of phase merging algorithms (PMA) it is assumed below that $\lambda_{v} \gg \lambda_{d}$ and $\mu_{v} \gg \mu_{d}$. This assumption is not extraordinary for an integrating voice/data $\mathrm{CWN}$, since this is a regime that commonly occurs in multimedia networks, in which wideband d-calls have both longer holding times and significantly smaller arrival rates than narrowband v-calls, e.g. see [16, 17]. Moreover, it is more important to note, that the final results as shown below, are independent of traffic parameters, and are determined from their ratio, i.e. the developed approach can provide a refined approximation even when parameters of heterogeneous traffic are only moderately distinctive.

The following splitting of state space (1) is examined:

$$
S=\bigcup_{k=0}^{N_{2}} S_{k}, \quad S_{k} \bigcap S_{k^{\prime}}=\varnothing, \quad k \neq k^{\prime},
$$

where $S_{k}:=\left\{\boldsymbol{n} \in S: n_{d}=k\right\}$.

Further state classes $S_{k}$ combine into separate merged states $\langle k\rangle$ and the following merging function in state space $S$ is introduced:

$$
U(\boldsymbol{n})=<k>\text { if } \boldsymbol{n} \in S_{k}, k=\overline{0, N_{2}} .
$$

Function (9) determines the merged model which is a one-dimensional Markov chain (1-D MC) with the state space $\tilde{S}:=\left\{\langle k\rangle: \quad k=\overline{0, N_{2}}\right\}$. Then, according to PMA, the stationary distribution of the initial model approximately equals:

$$
p(k, i) \approx \rho_{k}(i) \pi(<k>), \quad(k, i) \in S_{k}, \quad k=\overline{0, N_{2}},(10)
$$

where $\left\{\rho_{k}(i):(k, i) \in S_{k}\right\}$ is stationary distribution of a split model with state space $S_{k}$ and $\{\pi(<k>):<k>\in \tilde{S}\}$ is stationary distribution of a merged model, respectively.

By using (2) we conclude that the elements of generating matrix of this 1-D birth-death processes (BDP) $q_{k}(i, j)$ are obtained as follows:

$$
q_{k}(i, j)= \begin{cases}\lambda_{v} & \text { if } i \leq N_{3}-k-1, j=i+1, \\ \lambda_{h v} & \text { if } N_{3}-k \leq i<N, j=i+1, \\ i \mu_{v} & \text { if } j=i-1, \\ 0 & \text { in other cases. }\end{cases}
$$

So, stationary distribution within class $S_{k}$ is the same as that $M / M / N-k / N-k$ queuing system where the service rate of each channel is constant, $\mu_{v}$ and arrival rates are variable quantities. Hence

$$
\rho_{k}(i)= \begin{cases}\frac{v_{v}^{i}}{i !} \rho_{k}(0) & \text { if } 1 \leq i \leq N_{3}-k, \\ \left(\frac{v_{v}}{v_{h v}}\right)^{N_{3}-k} \frac{v_{h v}^{i}}{i !} \rho_{k}(0) & \text { if } N_{3}-k+1 \leq i \leq N-k,\end{cases}
$$

$$
\begin{aligned}
& \text { where } \rho_{k}(0)=\left(\sum_{i=0}^{N_{3}-k} \frac{v_{v}^{i}}{i !}+\left(\frac{v_{v}}{v_{h v}}\right)^{N_{3}-k} \sum_{i=N_{3}-k+1}^{N-k} \frac{v_{h v}^{i}}{i !}\right)^{-1} \text {, } \\
& v_{v}:=\lambda_{v} / \mu_{v}, v_{h v}:=\lambda_{h v} / \mu_{v} .
\end{aligned}
$$

Then, from (2) and (11) by means of PMA elements of generating matrix of a merged model $q\left(<k>,<k^{\prime}>\right)$, $<k>,<k^{\prime}>\in \tilde{S}$ are found:

$$
q\left(<k>,<k^{\prime}>\right)=\left\{\begin{array}{l}
\lambda_{d} \sum_{i=0}^{N_{1}-k-1} \rho_{k}(i)+\lambda_{h d} \sum_{i=N_{1}-1}^{N_{2}-k-1} \rho_{k}(i) \\
\text { if } 0 \leq k \leq N_{1}-1, k^{\prime}=k+1, \\
\lambda_{h d} \sum_{i=0}^{N_{2}-k-1} \rho_{k}(i) \\
\text { if } N_{1} \leq k \leq N_{2}-1, k^{\prime}=k+1, \\
k \mu_{d} \\
\text { if } k^{\prime}=k-1, \\
0 \\
\text { in other cases. }
\end{array}\right.
$$

The latter formula allows determining the stationary distribution of a merged model. It coincides with an appropriate distribution of state probabilities of a 1-D BDP, for which transition intensities are determined in accordance with (12). Consequently, stationary distribution of a merged model is determined as

$$
\pi(<k>)=\frac{\pi(<0>)}{k ! \mu_{d}^{k}} \prod_{i=1}^{k} q(<k-1>,<k>), k=\overline{1, N_{2}},
$$

where, $\pi(<0>)=\left(1+\sum_{k=1}^{N_{2}} \frac{1}{k ! \mu_{d}^{k}} \prod_{i=1}^{k} q(<k-1>,<k>)\right)^{-1}$

Then by using (11) and (13) from (10) stationary distribution of the initial 2-D MC can be found. So, summarizing the above given and omitting the complex algebraic transformations the following approximate formulae for the calculation of QoS metrics (3)-(7) can be suggested:

$$
\begin{aligned}
P_{h v} & \approx \sum_{k=0}^{N_{2}} \pi(<k>) \rho_{k}(N-k) ; \\
P_{o v} & \approx \sum_{k=0}^{N_{2}} \pi(<k>) \sum_{i=N_{3}-k}^{N-k} \rho_{k}(i) ; \\
P_{h d} & \approx \sum_{k=0}^{N_{2}} \pi(<k>) \sum_{i=N_{2}-k}^{N-k} \rho_{k}(i) ; \\
P_{o d} & \approx \sum_{k=0}^{N_{1}-1} \pi(<k>) \sum_{i=N_{1}-k}^{N-k} \rho_{k}(i)+\sum_{k=N_{1}}^{N_{2}} \pi(<k>) ; \\
\tilde{N} & \approx \sum_{i=1}^{N} i \sum_{k=0}^{f_{N_{2}}(i)} \pi(<k>) \rho_{k}(i-k) .
\end{aligned}
$$


Hereinafter $f_{k}(x)= \begin{cases}x & \text { if } 1 \leq x \leq k, \\ k & \text { if } k \leq i \leq N\end{cases}$

Now we can develop the algorithm to calculate the QoS metrics of the investigated multi-parametric CAC for the similar model with wide-band d-calls (due to the limited volume of work this algorithm does not present here).

\section{The CAC Based on Threshold Strategy}

Now we consider an alternative CAC in the integrated voice/data networks which is based on a threshold strategy. A more detailed description of the given CAC, follows. As in the CAC based on guard channels, we assume that an arrived hv-call is accepted as long as at least one free channel is available; otherwise it is blocked. For the purpose of definition of CAC based on a threshold strategy for calls of other types, three parameters $R_{1}$, $R_{2}$ and $R_{3}$, where $1 \leq R_{1} \leq R_{2} \leq R_{3} \leq N$ are introduced. Then the proposed CAC defines the following rules for admission of heterogeneous calls: an od-call (respectively, hd-call and ov-call) is accepted only if the number of calls of the given type in progress is less than $R_{l}$ (respectively, $R_{2}$ and $R_{3}$ ) and a free channel is available; otherwise it is blocked.

For the sake of simplicity we shall assume that $b=1$. The case $b>1$ is straightforward (see Section 2). The state of the system under the given CAC at any time is also described by 2-D vector $\boldsymbol{n}=\left(n_{d}, n_{v}\right)$, where $n_{d}$ (respectively, $n_{\mathrm{v}}$ ) is the number of data (respectively, voice) calls in the channels. Then state space of appropriate 2-D $\mathrm{MC}$ is given by:

$$
S:=\left\{\boldsymbol{n}: n_{d}=\overline{0, R_{2}}, n_{v}=\overline{0, N} ; n_{d}+n_{v} \leq N\right\} .
$$

The elements of generating matrix of the appropriate 2-D MC in this case is determined as follows:

$$
q\left(\boldsymbol{n}, \boldsymbol{n}^{\prime}\right)= \begin{cases}\lambda_{d} & \text { if } n_{d} \leq R_{1}-1, \boldsymbol{n}^{\prime}=\boldsymbol{n}+\boldsymbol{e}_{1}, \\ \lambda_{h d} & \text { if } R_{1} \leq n_{d} \leq R_{2}-1, \boldsymbol{n}^{\prime}=\boldsymbol{n}+\boldsymbol{e}_{1}, \\ \lambda_{v} & \text { if } n_{v} \leq R_{3}-1, \boldsymbol{n}^{\prime}=\boldsymbol{n}+\boldsymbol{e}_{2}, \\ \lambda_{h v} & \text { if } R_{3} \leq n_{v} \leq N-1, \boldsymbol{n}^{\prime}=\boldsymbol{n}+\boldsymbol{e}_{2}, \\ n_{d} \mu_{d} & \text { if } \boldsymbol{n}^{\prime}=\boldsymbol{n}-\boldsymbol{e}_{1}, \\ n_{v} \mu_{v} & \text { if } \boldsymbol{n}^{\prime}=\boldsymbol{n}-\boldsymbol{e}_{2}, \\ 0 & \text { in other cases. }\end{cases}
$$

Blocking probability of hv-calls and the mean number of busy channels are defined similarly to (3) and (7), respectively. The other QoS metrics are defined as the following marginal distributions of initial chain:

$$
P_{o v}:=\sum_{n \in S} p(\boldsymbol{n}) I\left(n_{v} \geq R_{3}\right)
$$

$$
\begin{gathered}
P_{h d}:=\sum_{n \in S} p(\boldsymbol{n}) \delta\left(n_{d}, R_{2}\right)+\sum_{n \in S} p(\boldsymbol{n}) \delta\left(n_{d}+n_{v}, N\right) I\left(n_{d}<R_{2}\right), \\
P_{o d}:=\sum_{n \in S} p(\boldsymbol{n}) I\left(n_{d} \geq R_{1}\right)+\sum_{n \in S} p(\boldsymbol{n}) \delta\left(n_{d}+n_{v}, N\right) I\left(n_{d}<R_{1}\right) .
\end{gathered}
$$

Unlike the CAC based on the guard channel strategy, it is easy to see that under this one there is no circulation flow in the state diagram of the underlying 2-D MC, i.e., reversible [15]. In other words, there is a general solution to the system of local balance equations (SLBE) in this chain. Therefore, by choosing any path between these states in the state diagram, we can express any state probability $p\left(n_{d}, n_{v}\right)$ using the state probability $p(0,0)$. So, in case $R_{2}+R_{3} \leq N$ we get the following multiplicative solution for stationary distribution of the underlying 2-D MC:

$$
p\left(n_{d}, n_{v}\right)=\left\{\begin{array}{l}
\frac{v_{d}^{n_{d}}}{n_{d} !} \cdot \frac{v_{v}^{n_{v}}}{n_{v} !} \cdot p(0,0), \\
\text { if } n_{d} \leq R_{1}, n_{v} \leq R_{3}, \\
\frac{v_{d}^{n_{d}}}{n_{d} !} \cdot \frac{v_{h v}^{n_{v}}}{n_{v} !}\left(\frac{v_{v}}{v_{h v}}\right)^{R_{3}} \cdot p(0,0), \\
\text { if } n_{d} \leq R_{1}, R_{3}<n_{v} \leq N, \\
\frac{v_{h d}^{n_{d}}}{n_{d} !} \cdot \frac{v_{v}^{n_{v}}}{n_{v} !} \cdot\left(\frac{v_{d}}{v_{h d}}\right)^{R_{1}} \cdot p(0,0), \\
\text { if } R_{1}<n_{d} \leq R_{2}, n_{v} \leq R_{3}, \\
\frac{v_{h d}^{n_{d}}}{n_{d} !} \cdot \frac{v_{h v}^{n_{v}}}{n_{v} !} \cdot\left(\frac{v_{d}}{v_{h d}}\right)^{R_{1}} \cdot\left(\frac{v_{v}}{v_{h v}}\right)^{R_{3}} \cdot p(0,0), \\
\text { if } R_{1}<n_{d} \leq R_{2}, R_{3}<n_{v} \leq N,
\end{array}\right.
$$

where $p(0,0)$ is determined from normalizing condition,

$$
\begin{aligned}
p(0,0)= & \left(\sum_{n \in S_{1}} \frac{v_{d}^{n_{d}}}{n_{d} !} \cdot \frac{v_{v}^{n_{v}}}{n_{v} !}+\left(\frac{v_{v}}{v_{h v}}\right)^{R_{3}} \sum_{n \in S_{2}} \frac{v_{d}^{n_{d}}}{n_{d} !} \cdot \frac{v_{h v}^{n_{v}}}{n_{v} !}+\left(\frac{v_{d}}{v_{h d}}\right)^{R_{1}}\right. \\
& \left.\sum_{n \in T_{3}} \frac{v_{h d}^{n_{d}}}{n_{d} !} \cdot \frac{v_{v}^{n_{v}}}{n_{v}}+\left(\frac{v_{d}}{v_{h d}}\right)^{R_{1}}\left(\frac{v_{v}}{v_{h v}}\right)^{R 3} \sum_{n \in S_{2}} \frac{v_{h d}^{n_{d}}}{n_{d} !} \cdot \frac{v_{h v}^{n_{v}}}{n_{v} !}\right)^{-1}
\end{aligned}
$$

Here we use the following notations: $v_{d}:=\lambda_{d} / \mu_{d}, v_{h d}$ : $=\lambda_{h d} / \mu_{d}$;

$$
\begin{aligned}
& S_{1}:=\left\{\boldsymbol{n} \in S: n_{d} \leq R_{1}, n_{v} \leq R_{3}\right\}, \\
& S_{2}:=\left\{\boldsymbol{n} \in S: n_{d} \leq R_{1}, R_{3}+1 \leq n_{v} \leq N\right\}, \\
& S_{3}:=\left\{\boldsymbol{n} \in S: R_{1}+1 \leq n_{d} \leq R_{2}, n_{v} \leq R_{3}\right\}, \\
& S_{4}:=\left\{\boldsymbol{n} \in S: R_{1}+1 \leq n_{d} \leq R_{2}, R_{3}+1 \leq n_{v} \leq N\right\} .
\end{aligned}
$$

In the case $R_{2}+R_{3}>N$ stationary distribution has the following form: 


$$
p\left(n_{d}, n_{v}\right)=\left\{\begin{array}{l}
\frac{v_{d}^{n_{d}}}{n_{d} !} \cdot \frac{v_{v}^{n_{v}}}{n_{v} !} \cdot p(0,0), \\
\text { if } 0 \leq n_{d} \leq R_{1}, 0 \leq n_{v} \leq R_{3}, \\
\frac{v_{h d}^{n_{d}}}{n_{d} !} \cdot \frac{v_{v}^{n_{v}}}{n_{v} !} \cdot\left(\frac{v_{d}}{v_{h d}}\right)^{R_{2}} \cdot p(0,0), \\
\text { if } R_{1}+1 \leq n_{d} \leq R_{2}, 0 \leq n_{v} \leq N-n_{d}, \\
\frac{v_{d}^{n_{d}}}{n_{d} !} \cdot \frac{v_{h v}^{n_{v}}}{n_{v} !} \cdot\left(\frac{v_{v}}{v_{h v}}\right)^{R_{3}} \cdot p(0,0), \\
\text { if } 0 \leq n_{d} \leq N-R_{3}-1, R_{3}+1 \leq n_{v} \leq N,
\end{array}\right.
$$

where

$$
\begin{aligned}
p(0,0)= & \left(\sum_{n \in T_{1}} \frac{v_{d}^{n_{d}}}{n_{d} !} \cdot \frac{v_{v}^{n_{v}}}{n_{v} !}+\left(\frac{v_{d}}{v_{h d}}\right)^{R_{1}} \sum_{n \in T_{2}} \frac{v_{h d}^{n_{d}}}{n_{d} !} \cdot \frac{v_{v}^{n_{v}}}{n_{v} !}+\left(\frac{v_{v}}{v_{h v}}\right)^{R_{3}}\right. \\
& \left.\sum_{n \in T_{3}} \frac{v_{d}^{n_{d}}}{n_{d} !} \cdot \frac{v_{h v}^{n_{v}}}{n_{v}}\right)^{-1} ; \\
T_{1}:= & \left\{\boldsymbol{n} \in S: 0 \leq n_{d} \leq R_{1}, 0 \leq n_{v} \leq R_{3}\right\} \\
T_{2}:= & \left\{\boldsymbol{n} \in S: R_{1}+1 \leq n_{d} \leq R_{2}, 0 \leq n_{v} \leq N-n_{d}\right\}, \\
T_{3}:= & \left\{\boldsymbol{n} \in S: 0 \leq n_{d} \leq N-R_{3}-1, R_{3}+1 \leq n_{v} \leq N\right\}
\end{aligned}
$$

The exact method to determine the steady state probabilities, in terms of a multiplicative representation (25) (or (26)) for large values of $N$, encounters numerical problems such as imprecision and overflow. These are related to the fact that with such a method the entire state space has to be generated, and large factorials and powers, close to zero, of the quantities (for low loads) or large values (for high loads) have to be calculated, i.e. there arises the problem of exponent overflow or underflow. Hence we can use a developed approximate method to determine the QoS metrics of the model, under the use of the proposed CAC based on threshold strategy, even when state space (19) is large.

As in Section 2, we assume that $\lambda_{v} \gg \lambda_{d}$ and $\mu_{v} \gg \mu_{d}$ and examine the following splitting of the state space

$$
S=\bigcup_{k=0}^{R_{2}} S_{k}, \quad S_{k} \bigcap S_{k^{\prime}}=\varnothing, \quad k \neq k^{\prime},
$$

where $S_{k}:=\left\{\boldsymbol{n} \in S: n_{d}=k\right\}$.

Next classes of states $S_{k}$ are combined into individual merged states $\langle k>$ and in (19) the merged function with range $\tilde{S}:=\left\{\langle k\rangle: \quad k=0,1, \cdots, R_{2}\right\}$ which is similar to (27) is introduced. As in the exact algorithm, in order to find the stationary distribution within splitting classes $S_{k}$ we will distinguish two cases: 1) $R_{2}+R_{3} \leq N$ and 2) $R_{2}+R_{3}$ $>N$. In the first case, the elements of the generating matrix, of appropriate 1-D BDP, are the same for all splitting models, i.e.

$$
q_{k}(i, j)= \begin{cases}\lambda_{v} & \text { if } i \leq R_{3}-1, j=i+1, \\ \lambda_{h v} & \text { if } R_{3} \leq i \leq N-1, j=i+1, \\ i \mu_{v} & \text { if } j=i-1, \\ 0 & \text { in other cases. }\end{cases}
$$

From the last formula we conclude that the stationary distribution within class $S_{k}$ is the same as that of the $M / M / N-k / N-k$ queuing system with state-dependent arrival rates and constant service rate of each channel, i.e.

$$
\rho_{k}(i)= \begin{cases}\frac{v_{v}^{i}}{i !} \rho_{k}(0) & \text { if } 1 \leq i \leq R_{3}, \\ \left(\frac{v_{v}}{v_{h v}}\right)^{R_{3}} \frac{v_{h v}^{i}}{i !} \rho_{k}(0) & \text { if } R_{3}+1 \leq i \leq N-k,\end{cases}
$$

where $\rho_{k}(0)=\left(\sum_{i=0}^{R_{3}} \frac{v_{v}^{i}}{i !}+\left(\frac{v_{v}}{v_{h v}}\right)^{R_{3}} \sum_{i=R_{3}+1}^{N-k} \frac{v_{h v}^{i}}{i !}\right)^{-1}$.

So, from (20) and (26) we conclude that elements of the generating matrix, of the merged model, are

$$
q\left(<k>,<k^{\prime}>\right)=\left\{\begin{array}{l}
\lambda_{d}\left(1-\rho_{k}(N-k)\right) \\
\text { if } 0 \leq k \leq R_{1}-1, k^{\prime}=k+1, \\
\lambda_{h d}\left(1-\rho_{k}(N-k)\right) \\
\text { if } R_{1} \leq k \leq R_{2}-1, k^{\prime}=k+1, \\
k \mu_{d} \\
\text { if } k^{\prime}=k-1, \\
0 \\
\text { in other cases. }
\end{array}\right.
$$

Distribution of the merged model is calculated by using (27) and has the following form:

$$
\pi(<k>)=\frac{\pi(<0>)}{k ! \mu_{d}^{k}} \prod_{i=1}^{k} q(<k-1>,<k>), k=\overline{1, R_{2}},
$$

where, $\pi(<0>)=\left(1+\sum_{k=1}^{R_{2}} \frac{1}{k ! \mu_{d}^{k}} \prod_{i=1}^{k} q(<k-1>,<k>)\right)^{-1}$.

Finally the following approximate formulae to calculate the desired QoS metrics, under the use of the proposed CAC based on the threshold strategy, are obtained:

$$
\begin{aligned}
P_{h v} & \approx \sum_{k=0}^{R_{2}} \pi(<k>) \rho_{k}(N-k) ; \\
P_{o v} & \approx \sum_{k=0}^{R_{2}} \pi(<k>) \sum_{i=R_{3}}^{N-k} \rho_{k}(i) ; \\
P_{h d} & \approx \pi\left(<R_{2}>\right)+\sum_{k=0}^{R_{2}-1} \pi(<k>) \rho_{k}(N-k) ;
\end{aligned}
$$




$$
\begin{aligned}
& P_{o d} \approx \sum_{k=R_{1}}^{R_{2}} \pi(<k>)+\sum_{k=0}^{R_{1}-1} \pi(<k>) \rho_{k}(N-k) ; \\
& N_{a v} \approx \sum_{k=1}^{N} k \sum_{i=0}^{f_{R_{2}}(k)} \pi(<i>) \rho_{i}(k-i) .
\end{aligned}
$$

In the second case (i.e. when $R_{2}+R_{3}>N$ ) distributions for splitting models with state space $S_{k}$ for $k=0,1$, $\cdots, N-R_{3}-1$ are calculated by using relations (26) while distributions for splitting models with state space $S_{k}$ for $k$ $=N-R_{3}, \cdots, R_{2}$ coincides with distributions of model $M / M / N-k / N-k$ with load $v_{v}$ erl, see (22). And all stages of the developed procedure, to calculate the QoS metrics, are the same as in the first case except the calculating of $P_{o v}$. The last QoS metric in this case is calculated as follows:

$$
P_{o v} \approx \sum_{k=0}^{N-R_{3}} \pi(<k>) \sum_{i=R_{3}}^{N-k} \rho_{k}(i)+\sum_{k=N=R_{3}+1}^{R_{2}} \pi(<k>) \rho_{k}(N-k) .
$$

\section{Numerical Results}

First briefly consider some results for the CAC based on the guard channels strategy in the integrated voice/data model with four classes of calls. The developed approximate formulas allow, without any computing difficulties, to carry out the authentic analysis of the QoS metrics in any change of the values of the loading parameters in the heterogeneous traffic, satisfying the assumption concerning their ratio (i.e. when $\lambda_{v} \gg \lambda_{d}$ and $\mu_{v} \gg \mu_{d}$ ) and also at any number of channels in a cell. Some results are shown in Figures 1-3 where $N=16, N_{3}$ $=14, N_{2}=10, \lambda_{o v}=10, \lambda_{h v}=6, \lambda_{o d}=4, \lambda_{h d}=3, \mu_{v}=10$, $\mu_{d}=2$. Behavior of the studied curves fully confirms all theoretical expectations.

In the given model, at the fixed value of the total number of channels $(N)$, it is possible to change values of three threshold parameters $\left(N_{1}, N_{2}\right.$ and $\left.N_{3}\right)$. In other words, there is three degrees of freedom. Note, that the increase in value of one of the parameters (in admissible area) favorably influences the blocking of the probability of calls of the corresponding type only (see Figures 1 and 2). So, in these experiments, the increase in value of the parameter $N_{1}$ leads to a reduction of the blocking probability of od-calls, but other blocking probabilities (i.e. $P_{h v}, P_{o v}$ and $P_{h d}$ ) increase. At the same time, the increase in value of any parameter leads to an increase in the overall channels utilization (see Figure 3).

Research in other directions consists of an estimation of the accuracy of the developed approximate formulas to calculate the QoS metrics. Exact values (EV) of QoS metrics are determined from SGBE. It is important to note, that under fulfilling the mentioned assumptions related to the ratio of the loading parameters of hetero

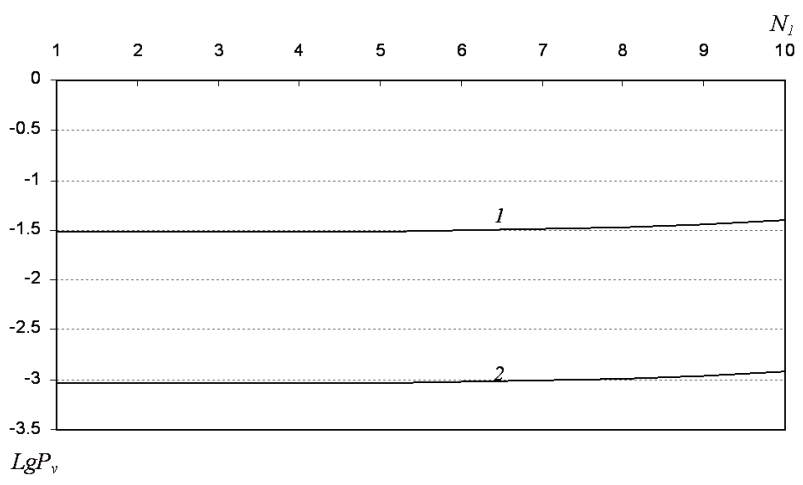

Figure 1. Blocking probability of v-calls versus $N_{1}: 1-P_{o v}$; 2-P $h$.

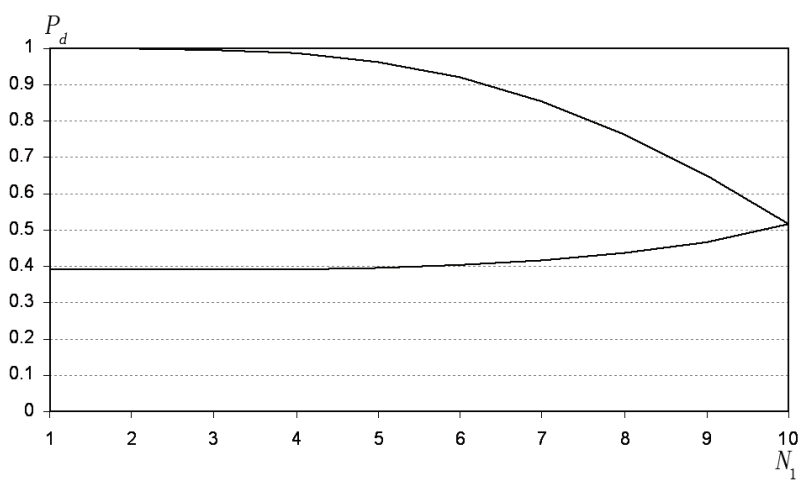

Figure 2. Blocking probability of d-calls versus $N_{1}: 1-P_{o d}$; 2-P $\boldsymbol{P}_{h d}$

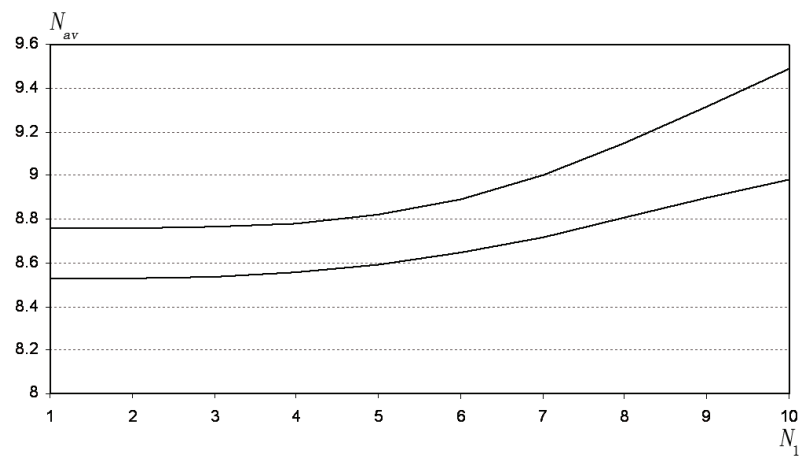

Figure 3. Average number of busy channels versus $N_{1}: 1-N_{3}$ $=15 ; 2-N_{3}=11$.

geneous traffic, the exact and approximate values (AV) almost completely coincide in all QoS metrics. Therefore these comparisons are not shown here. At the same time, it is obvious that finding the exact values of QoS metrics on the basis of the solution of SGBE appears effective only for models with moderate dimension.

It is important to note the sufficiently high accuracy of the suggested formulae even in the case where the accepted assumption about the ratio of the traffic loads is not fulfilled. To facilitate the computation efforts, as exact values of QoS metrics, we use their values calcu- 
lated from explicit formulas, see [21]. In mentioned work, appropriate results are obtained in the special case $b=1$ and $\mu_{v}=\mu_{d}$. Let's note, that condition $\mu_{v}=\mu_{d}$ contradicts our assumption $\mu_{v} \gg \mu_{d}$. Highest accuracy of the developed approximate formulas is observed at the calculation of the QoS metric for v-calls, since the maximal difference between exact and approximate values does not exceed 0.001 . Small deviations take place in the calculation of the QoS metrics for d-calls, but also thus in the worst case scenario the absolute error of the proposed formulas does not exceed 0.09 , that are quite comprehensible in an engineering practice. Similar results are observed for an average number of occupied channels of a cell. It is important to note, that numerous numerical experiments have shown, that at all admissible loads, the accuracy of the proposed approximate formulas grows with the increase in the value of the total number of channels. It is clear that in terms of simplicity and efficiency, the proposed approach is emphatically superior to the approach based on the use of balance equations to calculate the QoS metrics of the given CAC in the model with a non-identical channel occupancy time.

Also note, that high accuracy in the calculation of the QoS metrics for v-calls is observed even at those loadings which do not satisfy any of the accepted assumptions above concerning the ratio of intensities of heterogeneous traffic. So, for example, at the same values of number of channels and parameters of strategy, at $\lambda_{o v}=4$, $\lambda_{h v}=3, \lambda_{o d}=10, \lambda_{h d}=6, \mu_{v}=\mu_{d}=2$ (i.e. when assumptions $\lambda_{v} \gg \lambda_{d}, \mu_{v} \gg \mu_{d}$ are not fulfilled) the absolute error for the mentioned QoS metric does not exceed 0.002. Similar results are observed for an average number of occupied channels of a cell. However, the proposed approximate formulas show low accuracy for d-calls since for them the maximal absolute error exceeds 0.2 .

Numerical experiments with the CAC based on the threshold strategy are carried out also. Due to the limited volume of work these results are not presented here. As in CAC based on guard channels, the increase in value of one of the parameters (in admissible area), favorably affect the blocking probability of calls of the corresponding type only. So, the increase in value of parameter $R_{1}$ leads to a reduction of the blocking probability of od-calls but other blocking probabilities (i.e. $P_{h v}, P_{o v}$ and $\left.P_{h d}\right)$ increase. At the same time, the increase in the value of any parameter leads to an increase in the overall channels utilization.

At the end of this section we conducted research on comparative analysis of QoS metrics of two schemes: CAC based on the guard channels scheme and the CAC based on the threshold strategy. Comparison was done in the broad range of the number of channels and load parameters. In each access strategy the total number of channels is fixed and controllable parameters are $N_{1}, N_{2}$, $N_{3}$ (for CAC based on the guard channels scheme) and $R_{1}$,
$R_{2}, R_{3}$ (for CAC based on the threshold strategy). As mentioned above, the behavior of the QoS metrics, with respect to the indicated controllable parameters, in different CAC, are the same.

It is important to note that with the given number of channels, loads and QoS requirements in either of the CAC strategies may or may not meet the requirements. For instance, in the model of mono-service $\mathrm{CWN}$ for the given values of $N=100, v_{o}=50 \mathrm{erl}, v_{h}=35$ erl following requirements $P_{o} \leq 0.1, P_{h} \leq 0.007$ and $\tilde{N} \geq 80$ are not met with CAC based on guard channels irrespective of the value of the parameter $g$ (number of guard channels), whereas CAC based on individual pool only for h-calls (i.e. $r_{o}=0$ ) meets the requirements at $r_{h}=40$. However, for the same given initial data, requirements $P_{o} \leq 0.3, P_{h}$ $\leq 0.0001$ and $\tilde{N} \geq 60$ are only met by CAC based on the guard channel scheme at $g=20$, and never met by CAC based on the individual pool strategy irrespective of the value of its parameter $r_{h}$. Thus it is possible to find the optimal strategy (in a given context) at the given loads without changing the number of channels.

Apparently, both strategies have the same implementation complexity. That is why the selection of either of them, at each particular case, must be based on the answer to the following question: does it meet the given QoS requirements? These issues are a subject to a separate investigation.

\section{Conclusions}

In this paper, an effective and refined approximate approach to the performance analysis of the un-buffered integrated voice/data CWN, under different multiparametric $\mathrm{CAC}$, has been proposed. Note that many well-known results related to the mono-service $\mathrm{CWN}$ are special cases of such proposed ones. In almost all of the available work on devoted mono-service CWN, the queuing model is investigated with assumption that both handover and original calls are identical in terms of channel occupancy time. This assumption is rather limiting and unrealistic. Here, models of the un-buffered integrated voice/data CWN are explored with more general parameter requirements. Performed numerical results demonstrate high accuracy of the developed approximate method.

It is important to note that the proposed approach may facilitate the solution of problems related to the selection of the optimal (in given sense) values of parameters in the investigated multi-parametric CAC. These problems are subjects to separate investigation.

\section{Acknowledgements}

This research has been supported by Sangji University Research Fund 2009. 


\section{References}

[1] S. Tekinay and B. Jabbari, "Handover policies and channel assignment strategies in mobile cellular networks," IEEE Communication Magazine, Vol. 29, No. 11, pp. 42-46, 1991.

[2] I. Katzela and M. Naghshineh, "Channel assignment schemes for cellular mobile telecommunication systems," IEEE Personal Communications, pp. 10-31, June 1996.

[3] S. DasBit and S. Mitra, "Challenges of computing in mobile cellular environment-a survey," Computer Communications, Vol. 26, pp. 2090-2105, 2003.

[4] R. J. Boucherie and M. Mandjes, "Estimation of performance measures for product form cellular mobile communications networks," Telecommunication Systems, Vol. 10, pp. 321-354, 1998.

[5] R. J. Boucherie and N. M. Van Dijk, "On a queuing network model for cellular mobile telecommunications networks," Operation Research, Vol. 48, No. 1, pp. 38-49, 2000.

[6] W. Li and X. Chao, "Modeling and performance evaluation of cellular mobile networks," IEEE/ACM Transactions on Networking, Vol. 12, No. 1, pp. 131-145, 2004.

[7] D. Hong and S. S. Rapoport, "Traffic model and performance analysis of cellular mobile radio telephones systems with prioritized and non-prioritized handoff procedures," IEEE Transactions on Vehicular Technology, Vol. 35, No. 3, pp. 77-92, 1986.

[8] G. Haring, R. Marie, R. Puigjaner, and K. Trivedi, "Loss formulas and their application to optimization for cellular networks," IEEE Transactions on Vehicular Technology, Vol. 50, No. 3, pp. 664-673, 2001.

[9] B. Gavish and S. Sridhar, "Threshold priority policy for channel assignment in cellular networks," IEEE Transactions on Computers, Vol. 46, No. 3, pp. 367-370, 1997.

[10] V. S. Korolyuk and V. V. Korolyuk, "Stochastic model of systems," Kluwer Academic Publishers, Boston, 1999.

[11] W. Yue and Y. Matsumoto, "Performance analysis of multi-channel and multi-traffic on wireless communica- tion networks," Kluwer Academic Publishers, Boston, 2002.

[12] S. Nanda, "Teletraffic models for urban and suburban microcells: Cell sizes and hand-off rates," IEEE Transactions on Vehicular Technology, Vol. 42, No. 4, pp. 673$682,1993$.

[13] S. E. Ogbonmwan and L. Wei, "Multi-threshold bandwidth reservation scheme of an integrated voice/data wireless network," Computer Communications, Vol. 29, No. 9, pp. 1504-1515, 2006.

[14] R. W. Wolff, "Poisson arrivals see time averages," Operations Research, Vol. 30, No. 2, pp. 223-231, 1992.

[15] F. P. Kelly, "Reversibility and stochastic networks," John Wiley \& Sons, New York, 1979.

[16] V. Casares-Giner, "Integration of dispatch and interconnect traffic in a land mobile trunking system. Waiting time distributions," Telecommunication Systems, Vol. 16, No. 3-4, pp. 539-554, 2001.

[17] A. G. Greenberg, R. Srikant, and W. Whitt, "Resource sharing for book-ahead and instantaneous-request calls," IEEE/ACM Transactions on Networking, Vol. 7, No. 1, pp. 10-22, 1999.

[18] A. Z. Melikov and A. T. Babayev, "Refined approximations for performance analysis and optimization of queuing model with guard channels for handovers in cellular networks," Computer Communications, Vol. 29, No. 9, pp. 1386-1392, 2006.

[19] R. L. Freeman, "Reference manual for telecommunications engineering," John Wiley \& Sons, New York, 1994.

[20] A. Z. Melikov, M. I. Fattakhova, and A. T. Babayev, "Investigation of cellular communication networks with private channels for service of handover calls," Automatic Control and Computer Sciences, Vol. 39, No. 3, pp 61- 69, 2005.

[21] H. Chen, L. Huang, S. Kumar, an d C. C. Kuo, "Radio resource management for multimedia QoS supports in wireless networks," Kluwer Academic Publishers, Boston, 2004. 